\title{
Aktuelles rund um Hoden-, Prostata- und Blasenkarzinom
}

- In der Vorweihnachtszeit fand das vierte urologische Symposium der Firma medac statt. Die Themen lauteten diesmal: Fortschritte in der Versorgung von Patienten mit Hoden-, Prostata- oder Blasenkarzinomen sowie Neues zur Interstitiellen Zystitis. Auch aktuelle berufspolitische Fragen wurden erörtert. Die Leitung hatte Prof. Mark Schrader, Ärztlicher Direktor der Urologischen Klinik der Universitätsklinik UIm.

Schrader berichtete vom „Zweitmeinungszentrum Hodentumor", dessen Ziel es ist, die Versorgungsqualität bei Erhalt der dezentralen Versorgungsstruktur zu verbessern. Auf dem Internet-Auftritt www.zmhodentumor.de können Ärzte den klinischen Primärdatensatz und den Therapieplan ihrer Hodentumor-Patienten melden und erhalten dann eine evidenzbasierte Zweitmeinung zur gewählten Therapiestrategie. In dem von der Deutschen Krebshilfe geförderten Projekt konnten bisher in rund $30 \%$ der Fälle Über- oder Untertherapien vermieden werden.

Prof. Andres Jan Schrader, Universitätsklinik Ulm, stellte wichtige Studienergebnisse zur Hormontherapie des fortgeschrittenen Prostatakarzinoms vor: Er erläuterte beispielsweise, dass die intermittierende Hormontherapie empfohlen werden sollte. Neue interessante Therapeutika wie Abiraterone stehen vor der Zulassung, und in der Chemotherapie wird wohl trotz der Einführung von Cabazitaxel das bewährte Docetaxel weiter Standard bleiben.

Beim Harnblasenkarzinom sind laut Prof. Christian Wülfing, Asklepios Klinik Altona, folgende Empfehlungen auszusprechen: Nach jeder transurethralen Resektion der Harnblase (TURB) ist eine Frühinstillation zu geben und bei pT1-Tumoren immer eine Nachresektion durchzuführen. In der medi- kamentösen Therapie des metastasierten Blasenkarzinoms bleibt Gemcitabin-Cisplatin der First-Line-Standard.

Für die Interstitielle Zystitis steht mit Uropol ${ }^{\circledR}$ eine neue, hochkonzentrierte, in Studien geprüfte Chondroitin-Lösung zur Instillation zur Verfügung, die auftretende Symptome lindert. Uropol ${ }^{\oplus}$ kann auch bei der hyperaktiven Blase sowie bei einer durch Chemo- und Radiotherapie geschädigten Blase eingesetzt werden.

Dr. Florian Jentzmik, Universitätsklinik UIm, referierte über Aufbau und Funktion von Prostatakarzinom-Zentren. Abschließend gab der neu gewählte Präsident des Berufsverbandes Deutscher Urologen, Dr. Axel Schroeder, Neumünster, Einblicke in aktuelle gesundheitsund berufspolitische Entwicklungen.

Nach Informationen von

medac, Wedel

\section{Senföle als Antibiotika-Alternative}

— Die antibakterielle Wirkung der Senföle aus Kapuzinerkresse und Meerrettichwurzel (ANGOCIN ${ }^{\circledR}$ Anti-Infekt N) ist bereits seit einigen Jahren durch mehrere In-vitro-Studien am Universitätsklinikum Freiburg belegt: Die Untersuchungen zeigen ein breites antibakterielles Wirkspektrum der Senföle gegenüber 13 klinisch relevanten bakteriellen Erregern, sogar gegen Problemkeime wie MRSA, Vancomycin-resistente Enterokokken oder Penicillin-resistente Pneumokokken. Da sich die Senföle im Körper vorwiegend in Harnblase und Lunge anreichern, sind sie vor allem bei bakteriellen Harnwegs- und Atemwegsinfektionen eine Alternative zu chemischen Antibiotika.
Eine der häufigsten Nebenwirkungen einer Antibiotikatherapie bei Harnwegsinfekten (HWI) der Frau ist die Vaginalmykose. Je länger die Antibiose dauert, um so größer ist das Risiko, an einem Scheidenpilz zu erkranken. Die Senföle dagegen haben ein breites antibakterielles Wirkspektrum, schädigen im Gegensatz zu Antibiotika aber die Vaginalflora nicht.

„Um bei der HWI-Therapie" eine Induktion von Vaginalmykosen zu vermeiden und darüber hinaus den zunehmenden Antibiotikaresistenzen entgegenzuwirken, stellen die Senföle hier eine sinnvolle und wirksame Alternative zu chemischen Antibiotika dar", so Urologe Dr. Andreas Lucas, Dietzenbach. Aufgrund ihrer guten Verträglichkeit sind sie auch zur Prophylaxe rezidivierender HWI gut geeignet. Auf der Online-Plattform www.my-cme.de können Ärzte an einer Fortbildung zum Thema pflanzliche Antibiotika teilnehmen. Für die Information der Patienten steht die Broschüre „Pflanzliche Antibiotika - die sanfte Heilung aus der Natur (Bestellung unter www.repha.de) sowie die Internetseite www. pflanzliche-antibiotika.de zur Verfügung. Übrigens: Eine Studie der Universität Gießen belegt, dass pflanzliche Senföle auch antiviral gegen das neue H1N1-Virus wirken.

Nach Informationen von

Repha, Langenhagen
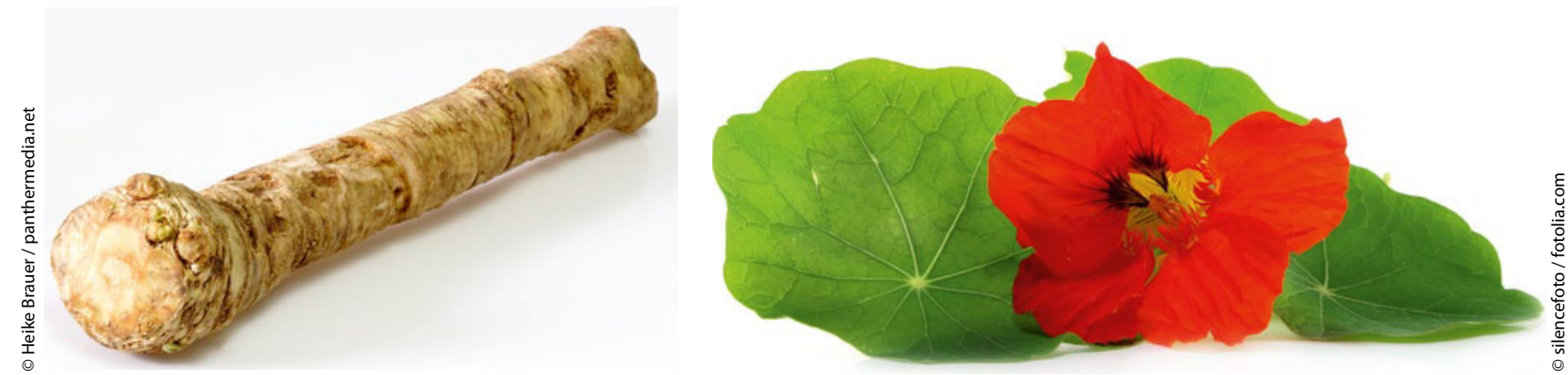

Senföle aus Meerrettichwurzel und Kapuzinerkresse als pflanzliche Antibiotika-Alternative 